\title{
Kognitive Verhaltenstherapie beugt erneuten Selbstverletzungen vor
}

Fragestellung: Dieses Cochrane-Review prüfte die Wirksamkeit von psychosozialen Interventionen bei selbstverletzendem Verhalten von Erwachsenen.

Hintergrund: Selbstverletzendes Verhalten ist insbesondere bei jungen Erwachsenen zwischen 15 und 35 Jahren verbreitet. Häufig geschieht es zum wiederholten Mal und ist mit Suiziden assoziiert.

Patienten und Methodik: In den elektronischen Datenbanken CCDANCTR-Studies and References, CENTRAL, MEDLINE, Embase und PsychINFO wurden für den Zeitraum zwischen 1998 und 2015 randomisierte kontrollierte Studien (RCT) zu psychosozialen Interventionen für Erwachsene nach selbstverletzendem Verhalten in den vergangenen sechs Monaten identifiziert. Berichtet werden Ergebnisse bezüglich Interventionen, zu denen mindestens drei Studien gehören, welche die Interven-

Hawton K, Witt KG, Salisbury TLT et al. Psychosocial interventions following self-harm in adults: a systematic review and meta-analysis. Lancet Psychiatry 2016; 3: 740-50 tion mit TAU (Treatment As Usual) vergleichen.

Als primärer Endpunkt war die Wiederholung des selbstverletzenden Verhaltens unmittelbar nach Therapieende sowie nach sechs, zwölf und 24 Monaten defi- niert. Sekundäre Endpunkte waren Häufigkeit des selbstverletzenden Verhaltens, Suizid, Suizidgedanken, Depression, Hoffnungslosigkeit und Problemlöseverhalten. Sofern vorhanden, wurden Intention-To-Treat(ITT)-Daten bevorzugt.

Ergebnisse: Die systematische Literaturrecherche identifizierte 29 RCT einschließlich drei unabhängiger Studien über dieselbe Intervention. Verhaltenstherapeutisch basierte Interventionen einschließlich kognitiver Verhaltenstherapie (KVT) und Problemlösetherapie konnten den Anteil der Teilnehmer, die erneut selbstverletzendes Verhalten zeigten, sechs Monate nach Therapieabschluss reduzieren (Odds Ratio 0,54), ebenso nach zwölf Monaten (Odds Ratio 0,80). Signifikante Verbesserungen ergaben sich auch in Bezug auf die sekundären Endpunkte Depression, Hoffnungslosigkeit, Suizidgedanken und Problemlöseverhalten.

Dialektisch-behaviorale Therapie (DBT) erzielte gegenüber TAU keinen signifikanten Effekt auf das Risiko wiederholten selbstverletzenden Verhaltens; eine Reduktion der Häufigkeit des Auftretens konnte jedoch aufgezeigt werden.

Schlussfolgerungen: KVT scheint das Auftreten von erneutem selbstverletzenden Verhalten zu verringern. Für DBT konnte eine Reduktion der Häufigkeit von selbstverletzendem Verhalten gezeigt werden.

\section{- Kommentar von Jessica Mattivi, Mainz}

\section{Verhaltenstherapien reduzieren selbstverletzendes Verhalten}

Bei der hier vorliegenden Arbeit handelt es sich um eine qualitativ hochwertige Übersichtsarbeit (systematischer Review und Metaanalyse) der Cochrane Collaboration. Die Ergebnisse zeigten, dass KVT die Wahrscheinlichkeit für das Auftreten von wiederholtem selbstverletzenden Verhalten reduziert und DBT die Episodenhäufigkeit solchen Verhaltens verringert. Einschränkend ist zu sagen, dass nur wenige Interventionsansätze in für eine Metaanalyse ausreichender Anzahl von Studien untersucht wurden.

„Postkarteninterventionen“ erzielten zwar in der Metaanalyse keine Effekte, nach Entfernung einer Pilotstudie mit geringer Fallzahl konnten aber signifikant positive Ergebnisse aufgezeigt werden. Diese wenig kostenintensive Interventionsart, bei der Patienten, die sich wegen selbstverletzenden Verhaltens in einer Einrichtung des Gesundheitssystems vorgestellt haben, in regelmäßigen Abständen Postkarten mit aufmunternden Botschaften erhalten, könnte insbesondere für ressourcenarme Umgebungen beziehungsweise in Regionen mit geringer Dichte von Psychotherapeuten eine Behandlungsoption darstellen.
Schließlich sollte bei der Interpretation der Ergebnisse des Reviews beachtet werden, dass in einem Drittel der eingeschlossenen Studien die Untersucher zusätzlich zu Therapeuten und Patienten - was in Psychotherapiestudien unvermeidlich ist - nicht verblindet waren, was das Risiko für einen Detection Bias erhöht.

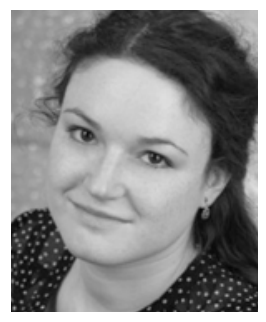

Dr. med. univ. Jessica Mattivi, Mainz

Klinik für Psychiatrie und Psychotherapie, Universitätsmedizin Mainz

E-Mail: jessica.mattivi@unimedizin-mainz.de 